\title{
Estrutura e organização trófica da ictiofauna de águas rasas em um estuário tropical
}

\author{
Andréa C. G. de Paiva '; Paulo de Tarso da C. Chaves ${ }^{2} \&$ Maria E. de Araújo ${ }^{1}$ \\ ${ }^{1}$ Laboratório de Nécton e Aqüicultura, Departamento de Oceanografia, Universidade Federal de Pernambuco. \\ Avenida Arquitetura, Cidade Universitária, 50740-550 Recife, Pernambuco, Brasil. \\ E-mail: acg_paiva@yahoo.com.br; betharau@terra.com.br \\ ${ }^{2}$ Laboratório de Ictiologia Estuarina, Departamento de Zoologia, Universidade Federal do Paraná. Caixa Postal 19020, \\ 81531-980 Curitiba, Paraná, Brasil. E-mail: ptchaves@ufpr.br
}

\begin{abstract}
The goals of the present study were: 1) to test whether the shallow water ichthyofaunal diversity in the Formoso river (Pernambuco) varies between the upper and lower estuarine zones and between the dry and rainy season; and 2) to assess spatial and temporal shifts in their trophic categories. The fishes were collected bimonthly using beach seine. A total of 5,475 individuals were analyzed, belonging to 78 species, of which $51.3 \%$ were of reef origin. Carangidae and Gerreidae had the highest diversity, whereas Clupeidae, Engraulidae and Gerreidae were the most abundant. Among the dominant species (89\%), Rhinosardinia amazonica was particularly common $(36 \%)$. Most of the species were predators $(75 \%)$, including species with carnivorous feeding habits of the $2^{\text {nd }}$ order (37.5\%). The only statistically significant difference in diversity occurred between the lower and upper estuarine zones, being higher in the former. This is probably due to the higher morphological complexity, to the larger proportion of Rhizophora mangle (shelter and refuge for several fishes), and to the mud sediment that makes this a more productive area. The Trophic Contribution Factor (FCT) for the debris was higher in the upper estuarine zone, where there is a higher rate of silt and clay. This TCF for primary production was higher in the middle estuarine zone, where there is higher phytoplankton biomass. Most species were in the young ecophase, corroborating the importance of shallow waters in this tropical estuary as a natural nursery and shelter for several fish of ecological and economical importance.
\end{abstract}

KEY WORDS. Ichtyophauna; feeding; mangroves; Pernambuco.

RESUMO. O presente estudo avaliou:1) se a diversidade da ictiofauna de águas rasas do Rio Formoso (Pernambuco) varia entre as zonas estuarinas superior e inferior, e entre os períodos seco e chuvoso e 2) se houve diferenças espaciais e temporais nas categorias tróficas dessa ictiofauna. Os peixes foram coletados bimestralmente com rede-de-arrasto tipo mangote. Foram coletados 5475 indivíduos pertencentes a 78 espécies, sendo $51,3 \%$ de origem recifal. Carangidae e Gerreidae tiveram a maior riqueza específica, enquanto Clupeidae, Engraulidae e Gerreidae a maior abundância. Entre as espécies dominantes (89\%), destaca-se Rhinosardinia amazonica (36\%). A maioria da ictiofauna é predadora (75\%) e com hábito alimentar carnívoro de $2^{\mathrm{a}}$. ordem $(37,5 \%)$. A diversidade foi significativamente mais elevada na zona superior do estuário. A zona superior do estuário é, provavelmente, mais produtiva devido a sua maior complexidade morfológica, à maior abundância de Rhizophora mangle (abrigo e refúgio para vários peixes) e ao sedimento lamoso. O Fator de Contribuição Trófica (FCT) de detritos foi maior na zona estuarina superior, onde há maior teor de silte e argila. O FTC para a produção primária foi mais elevado na zona estuarina média, onde há uma maior biomassa fitoplanctônica. A maioria das espécies encontrava-se em ecofase jovem, evidenciando a importância das águas rasas deste estuário tropical como criadouro natural e abrigo para vários peixes de importância ecológica e econômica.

PALAVRAS-CHAVE. Ictiofauna; alimentação; manguezais; Pernambuco.

A comunidade de peixes estuarinos é constituída por espécies residentes, migrantes marinhas e de água doce, que usam os estuários como áreas de alimentação, de criação de larvas e juvenis ou para a reprodução (BLABER 2000). Esses hábitats favorecem a presença de várias populações ícticas em suas mar- gens (VIDY 2000) constituídas principalmente por jovens de espécies marinhas (RozAs \& ZimmERMAn 2000). A abundância de peixes nos estuários deve-se principalmente à disponibilidade de alimentos, a partir da produção primária (ROBERTSON \& BLABER 1992); complexidade estrutural da vegetação de mangue, que 
propicia refúgio, principalmente para os peixes jovens; elevada turbidez da água; e reduzido número de peixes carnívoros de grande porte (Robertson \& Blaber 1992, Mullin 1995). A determinação da biodiversidade, especialmente da comunidade de peixes e dos seus padrões de variação espacial e temporal, é de grande relevância para avaliar a qualidade ambiental, uma vez que os peixes ocupam diversas posições na teia trófica (Teixeira et al. 2005).

Nos estuários, o hábito alimentar dos peixes é bastante diversificado, encontrando-se representantes de todas as categorias tróficas (CABERTY et al. 2004). De um modo geral, os herbívoros são representados por poucas espécies; os bentívoros são dominantes, e ambos ocorrem em todos os tipos de estuários. Os iliófagos e os planctívoros dominam nos estuários do tipo fechado e aberto, respectivamente; e os piscívoros são dominantes em estuários com influência de águas costeiras e abertos (Blaber 2000). A estrutura da ictiofauna estuarina tropical varia com o tipo de estuário (BLABER 2000) e com as diferenças no padrão espacial e temporal da comunidade (e.g. SPACH et al. 2003, 2004, Loebman \& Vieira 2005). O conhecimento da teia trófica auxilia na compreensão de tal estrutura e permite descrever o fluxo energético nos ecossistemas e as relações ecológicas entre os organismos (Almeida et al. 1997).

Estudos sobre alimentação em peixes normalmente restringem-se a descrições por espécie ou grupos de espécies. No Brasil os trabalhos abordando a organização trófica das comunidades são mais comuns em ambientes fluviais (e.g. RESENDE 2000, Hahn et al. 2004, Mérona \& Rankin-de-Mérona 2004) que na região costeira (e.g. Chaves \& Bouchereau 2004). Muitos desses estudos abordam a complexidade da alimentação dos peixes, porém não são multiespecíficos e não detectam as vias de fluxo de energia. A tendência é, então, descrever uma estrutura simplificada baseada em grupos tróficos de espécies dominantes (Livingston et al. 1986). A dinâmica trófica nos estuários tende a ser bastante complexa, uma vez que nesses ambientes existem duas teias, uma pastadora, tendo como base o fitoplâncton; e outra detritívora, com base nos detritos (DAy et al. 1989). A separação de espécies em grupos, de acordo com seus hábitos alimentares, é uma maneira de compreender a organização da comunidade, a relação presa-predador e a produção de biomassa (SoAres et al. 1993). Diante desse contexto o presente estudo visa responder às seguintes perguntas: 1) A diversidade da ictiofauna de águas rasas do Rio Formoso varia entre as zonas estuarinas superior, média e inferior e nos períodos seco e chuvoso? 2) Existem diferenças espaciais e temporais nas categorias tróficas da ictiofauna desse mesmo estuário?

\section{MATERIAL E MÉTODOS}

O presente estudo foi realizado no estuário do Rio Formoso, que nasce na porção noroeste do município de Rio Formoso $\left(08^{\circ} 37^{\prime}, 08^{\circ} 40^{\prime} \mathrm{S}\right.$ e $\left.35^{\circ} 04^{\prime}, 35^{\circ} 08^{\prime} \mathrm{W}\right)$ (CONDEPE 1992) e está inserido em duas Áreas de Proteção Ambiental (APAs), a APA Estadual de Guadalupe e APA Federal Costa dos Corais (Fig. 1).

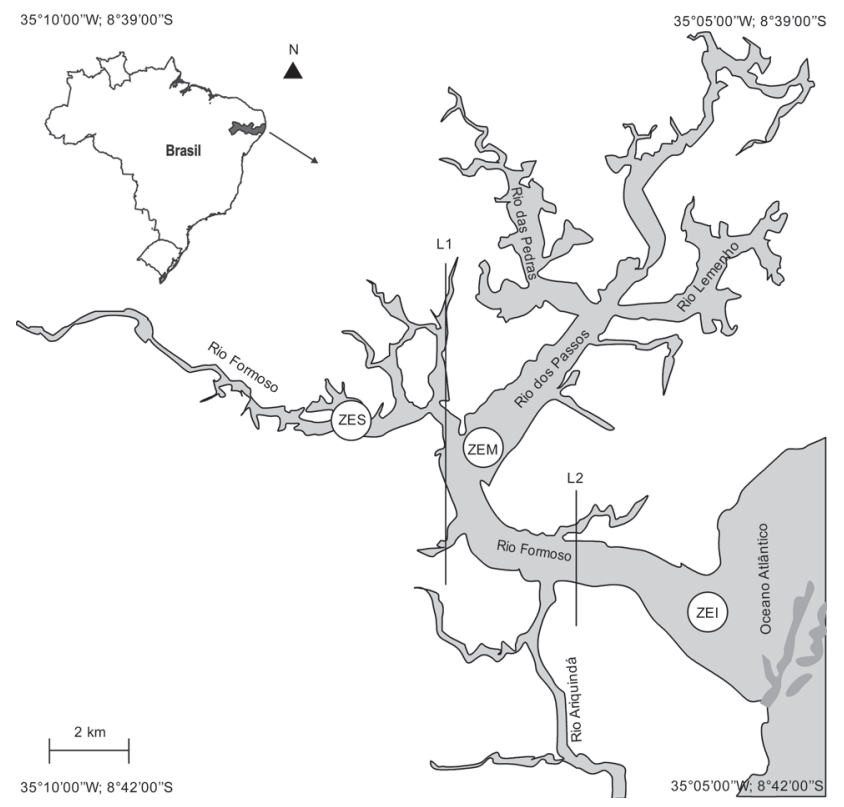

Figura 1. Mapa do estuário de Rio Formoso com a localização das áreas de amostragem: zona estuarina superior (ZES), média (ZEM) e inferior (ZEI). (L1) Limite entre as ZES e ZEM, (L2) limite entre as ZEM e ZEI, definidos por LIRA et al. (1979).

LiRA et al. (1979) sugeriram a divisão do estuário do Rio Formoso em três zonas morfologicamente distintas: estuarina superior, média e inferior. A primeira é formada por gamboas e canais de marés com margens muito colonizadas por manguezais; a segunda é menos colonizada por esse tipo de vegetação, apresentando trechos intercalados com coqueirais; e a zona estuarina inferior é caracterizada pela ausência de mangues.

O clima na região é do tipo As' no lado oeste, sendo caracterizado como quente e úmido, com chuvas de outono e inverno, e do tipo Ams' no leste (KöPPEN 1948), tropical chuvoso monção, com verão seco e precipitação pluviométrica anual de 2.000 $\mathrm{mm}$; apresentando temperatura média anual de $24^{\circ} \mathrm{C}$ (СРRH 1991).

Bimestralmente, entre outubro de 2005 e agosto de 2006 foram realizadas coletas diurnas nas zonas estuarinas. Foram efetuados três arrastos consecutivos, utilizando-se rede-de-arrasto tipo mangote $(22 \mathrm{~m} \times 2 \mathrm{~m}$ ) com malha de $8 \mathrm{~mm}$ entre nós adjacentes. A rede foi puxada manual e perpendicularmente à margem do estuário, em profundidades de $2,0 \mathrm{~m}$ a $0 \mathrm{~m}$ durante aproximadamente oito minutos.

Simultaneamente às coletas biológicas, foram tomados valores de temperatura e salinidade na água superficial através de termômetro com precisão de $1^{\circ} \mathrm{C}$ e refratômetro portátil (precisão de $1 \mathrm{ppm}$ ), respectivamente. Em cada zona foram coletadas amostras de água para a determinação em laboratório, do $\mathrm{pH}$ e teor oxigênio dissolvido $\left(\mathrm{ml}^{-1} \mathrm{l}^{-1}\right)$, este através da técnica de Winkler (Grasshoff et al. 1983). As amostras de sedimento para análise granulométrica (Suguio 1973) foram obtidas nos meses 
de dezembro e junho, correspondendo aos meses de período seco e chuvoso, respectivamente. A classificação do tipo de sedimento, baseada no teor de silte e argila, seguiu U.S. NAVAL Oceanographic Office (1965). Os dados mensais de precipitação pluviométrica, nos anos de 2005 e 2006, foram fornecidos pelo Instituto Nacional de Meteorologia (INMET).

Os peixes foram identificados, medidos e pesados. Para a identificação dos espécimes foram utilizadas principalmente as diagnoses de Araújo et al. (2004), Figueiredo \& Menezes (1978, 1980, 2000), Cervigón (1991, 1993, 1994, 1996), Menezes (1983), Menezes \& Figueiredo (1980, 1985), seguindo a ordenação filogenética citada em Nelson (1994). Os espécimes estão depositados na Coleção Ictiológica do Departamento de Oceanografia, Universidade Federal de Pernambuco, sob o número de tombamento 1390 a 1476.

Para classificar as espécies quanto à abundância e à freqüência de ocorrência foi utilizada metodologia adaptada de Garcia \& Vieira (2001), sendo consideradas abundantes as espécies que tiveram captura percentual (PN\%) maior que a razão 100/S, onde S é o número de espécies; e freqüentes àquelas aquelas que tiveram freqüência de ocorrência (FO\%) maior que $50 \%$. A partir daí, as espécies foram classificadas e agrupadas em função dos seus valores de PN\% e FO\% em: pouco abundantes e pouco freqüentes (PA-PF), (PN\% < 100/S e FO\% $<50 \%$ ); pouco abundantes e freqüentes (PA-F), (PN\% < 100/S e $50=\mathrm{FO} \%(86,6 \%)$; pouco abundantes e muito freqüentes (PA$\mathrm{MF}),(\mathrm{PN} \%<100 / \mathrm{S}$ e $\mathrm{FO} \%(86,6 \%)$; abundantes e pouco freqüentes (A-PF), (PN\% > 100/S e FO\% < 50\%); abundantes e freqüentes (A-F), (PN\% > 100/S e FO\% > 50\%) e abundantes e muito freqüentes (PN\% > 100/S e FO\%>86,6\%).

Para a análise da distribuição espacial foram consideradas as espécies abundantes e muito freqüentes (A-MF) e abundantes e freqüentes (A-F) nas zonas estuarinas. Essas espécies foram classificadas em três grupos: G1, espécies A-MF e A-F nas zonas estuarinas superior e média; G2, espécies A-MF e A-F nas zonas média e inferior; e G3, que corresponde às espécies A-MF e A-F em todas as zonas estuarinas. Para a análise da distribuição temporal também foram consideradas as espécies A-MF e A-F nos períodos seco (setembro a fevereiro) e chuvoso (março a agosto) (MACÊDo et al. 2000).

A diversidade da ictiofauna foi calculada com base no índice de Shannon-Wiener $\left(H^{\prime}=a ̊\right.$ pi $\log _{2}$ pi) através do programa Primer 5.1.2. Para testar se houve diferenças na diversidade e categorias tróficas, entre as zonas estuarinas e entre os meses dos períodos seco e chuvoso, foi aplicada a Análise de Variância (ANOVA: dois critérios), exceto para as categorias tróficas nos períodos seco e chuvoso, onde foi utilizado o teste $t$ Student para dados independentes. Para estas análises foram realizados os testes de normalidade Shapiro-Wilk e de homocedasticidade de Cochran. Nas análises em que ocorreu significância estatística, foi utilizado o teste de Tukey para a comparação das médias. Os testes mencionados acima foram realizados no programa Bioestat 4.0 utilizando nível de significância de 5\%.
O hábito alimentar das espécies foi descrito através de informações obtidas na literatura, sendo a classificação das categorias tróficas adaptada de BouchON-Navaro et al. (1992): herbívoros $(\mathrm{H})$, peixes que consomem desde algas até fanerógamas marinhas; planctívoros $(\mathrm{P})$; onívoros $(\mathrm{O})$, que ingerem invertebrados e algas; carnívoros de primeira ordem (CI) consomem preferencialmente pequenos invertebrados bentônicos; carnívoros de segunda ordem (CII) consomem principalmente invertebrados e peixes; e carnívoros de terceira ordem (CIII), cuja alimentação é constituída em mais de $80 \%$ por peixes. Adicionou-se a essa classificação a categoria iliófago-detritívoro (ID) (ZaVAla-CAMIN 1996). A seguir estimou-se a abundância relativa de peixes de cada categoria segundo a zona estuarina e o período, seco ou chuvoso. Através do Fator de Contribuição Trófica (FCT), adaptado de Chaves \& Bouchereau (2004), foram comparadas as categorias tróficas nas zonas estuarinas superior, média e inferior; e entre os períodos seco e chuvoso: $\mathrm{FCTs}_{\mathrm{i}}=\Sigma_{\mathrm{cl} \mapsto \mathrm{cVl}}\left(\mathrm{B}_{\mathrm{i} . \mathrm{c} . \mathrm{c}} \mathrm{F}_{\mathrm{c}}\right) /\left(\Sigma_{\mathrm{il} \mapsto \mathrm{in}} \Sigma_{\mathrm{cVl}}\left(\mathrm{B}_{\mathrm{i} . \mathrm{c} .} \mathrm{F}_{\mathrm{c}}\right)\right.$, onde $\mathrm{i}=$ período do ano (seco: outubro, dezembro e fevereiro; chuvoso: abril, junho e agosto); $\mathrm{n}=$ número de unidades das variáveis ( 3 para as zonas estuarinas e 2 para os períodos do ano); $\mathrm{B}=$ biomassa nas categorias tróficas (c); e $\mathrm{F}$ = fator de uso trófico em cada categoria trófica (detrito ou produção primária). Foram atribuídos os valores 0 , 1, 2, 3 ou 4 para os graus crescentes de contribuição direta da fonte inicial de energia para uma determinada categoria trófica. Quando os membros de uma categoria tinham uma ligação muito próxima com os detritos ou produção primária, o valor atribuído para $\mathrm{F}$ é 4 . Ao contrário, quando a categoria trófica não era diretamente ligada aos detritos ou produção primária, o valor de F é 0. O FCT para as fontes de detritos $(\mathrm{s}=\mathrm{d})$ ou produção primária $(\mathrm{s}=\mathrm{pp})$ combinou a biomassa das espécies de cada categoria trófica e o fator para a fonte inicial de energia (d ou pp) para cada categoria trófica.

\section{RESULTADOS}

De um modo geral, os maiores valores médios de temperatura, salinidade, $\mathrm{pH}$ e oxigênio dissolvido ocorreram na zona estuarina inferior, e nos meses mais secos (Tab. I). O maior valor percentual de silte e argila no sedimento ocorreu na zona estuarina superior, seguido pela zona estuarina média (Fig. 2).

Foram coletados 5475 indivíduos pertencentes a 78 espécies e 39 famílias, sendo 51,3\% das espécies, de origem recifal. Carangidae e Gerreidae apresentaram a maior riqueza específica, ambas com seis espécies. Clupeidae $(\mathrm{n}=2333)$, Engraulidae ( $\mathrm{n}=1187)$ e Gerreidae $(\mathrm{n}=1145)$ foram as mais abundantes, contribuindo com $37,2 \%, 18,9 \%$ e $18,3 \%$ do número total de peixes coletados, respectivamente. Das 78 espécies, 12 foram classificadas como abundantes e muitos freqüentes (A-MF); uma, como abundante e freqüente (A-F); 10 espécies foram pouco abundantes, porém muito freqüentes (PA-MF); 18 foram pouco abundantes, mas freqüentes (PA-F); e 37, pouco abundantes e pouco freqüentes (PA-PF) (Tab. II). As espécies numericamente dominantes (A-MF ou A-F) representaram 
$89,06 \%$ do número total de indivíduos coletados, destacandose R. amazonica (Steindachner, 1879) (35,68\%) (Fig. 3).

Espacialmente, as espécies classificadas como abundantes e muito freqüentes ou freqüentes estiveram distribuídas entre as zonas superior e média (G1), média e inferior (G2) e em todas as zonas (G3) (Fig. 4).

Entre os meses dos períodos seco e chuvoso, o maior nú- mero de espécimes (2.982) ocorreu no período chuvoso, sendo 2.493 no período seco. Das espécies abundantes e muito freqüentes (A-MF) e abundantes e freqüentes (A-F), R. amazonica, C. edentulus (Cuvier, 1829) e C. spilopterus Günther, 1862 predominaram no período chuvoso; enquanto H. clupeola (Cuvier, 1829), E. brasilianus (Cuvier, 1830) e C. pectinatus (Poey, 1860), no período seco (Tab. III).
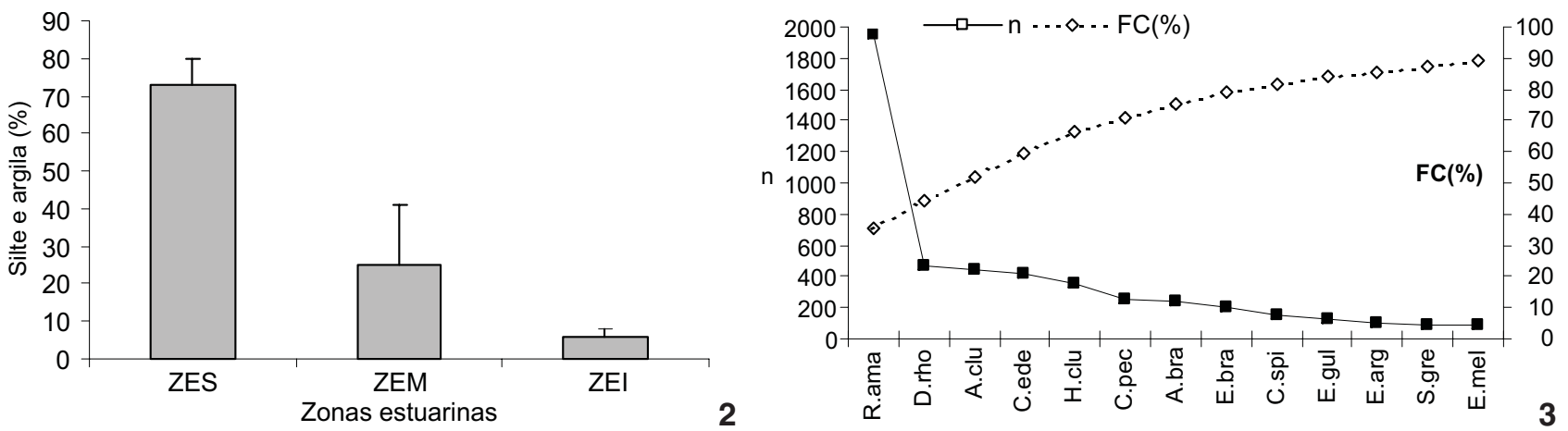

Figuras 2-3. (2) Valores percentuais médios de silte e argila nas zonas estuarinas superior (ZES), média (ZEM) e inferior (ZEI) do Rio Formoso. Barra vertical corresponde ao desvio padrão; (3) distribuição de abundância em número de indivíduos (n) e curva de freqüência acumulada (FC), em percentagem, das espécies abundantes e muito freqüentes ou freqüentes do estuário do Rio Formoso. (R.ama) Rhinosardinia amazonica, (D.rho) Diapterus rhombeus, (A.clu) Anchovia clupeoides, (C.ede) C. edentulus, (H.clu) H. clupeola, (C.pec) C. pectinatus, (A.bra) Atherinella brasiliensis, (E.bra) E. brasilianus, (C.spi) Citharichthys spilopterus, (E.gul) Eucinostomus gula, (E.arg) E. argenteus, (S.gre) S. greeley, (E.mel) E. melanopterus.

Tabela I. Valor médio (M) e desvio padrão (DP) da temperatura ( $T$ ), salinidade, pH e oxigênio dissolvido nas zonas estuarinas superior (ZES), média (ZEM) e inferior (ZEI) do Rio Formoso, nos meses de amostragem.

\begin{tabular}{|c|c|c|c|c|c|c|c|c|}
\hline \multirow{2}{*}{ Meses/Zonas } & \multicolumn{2}{|c|}{$\mathrm{T}\left({ }^{\circ} \mathrm{C}\right)$} & \multicolumn{2}{|c|}{$S$} & \multicolumn{2}{|c|}{$\mathrm{pH}$} & \multicolumn{2}{|c|}{$\mathrm{OD}\left(\mathrm{ml.} \mathrm{I}^{-1}\right)$} \\
\hline & M & DP & M & DP & M & DP & M & DP \\
\hline Out/ZES & 28,00 & 0,41 & 26,5 & 1,22 & 7,34 & 0,05 & 4,55 & 0,19 \\
\hline Out/ZEM & 27,67 & 0,24 & 35,0 & 1,47 & 7,48 & 0,09 & 3,85 & 0,23 \\
\hline Out/ZEI & 28,67 & 0,47 & 37,0 & 0,82 & 7,97 & 0,02 & 5,24 & 0,57 \\
\hline Dez/ZES & 29,67 & 0,47 & 34,0 & 0,82 & 8,12 & 0,01 & 4,31 & 0,27 \\
\hline Dez/ZEM & 28,33 & 0,62 & 36,0 & 1,08 & 8,25 & 0,01 & 3,94 & 0,19 \\
\hline Dez/ZEI & 28,17 & 0,24 & 36,0 & 0,41 & 8,30 & 0,10 & 4,83 & 0,24 \\
\hline Fev/ZES & 29,33 & 0,24 & 33,5 & 0,82 & 7,68 & 0,03 & 4,42 & 1,16 \\
\hline Fev/ZEM & 31,00 & 0,82 & 36,5 & 0,41 & 7,00 & 0,74 & 4,56 & 0,32 \\
\hline Fev/ZEI & 31,33 & 0,24 & 37,0 & 0,41 & 7,97 & 0,22 & 5,91 & 0,27 \\
\hline $\mathrm{Abr} / \mathrm{ZES}$ & 29,33 & 0,47 & 31,5 & 0,41 & 7,67 & 0,01 & 3,43 & 0,12 \\
\hline Abr/ZEM & 30,33 & 0,24 & 30,5 & 0,41 & 8,01 & 0,03 & 3,43 & 0,47 \\
\hline $\mathrm{Abr} / \mathrm{ZEI}$ & 30,50 & 0,82 & 37,5 & 0,41 & 8,06 & 0,38 & 4,65 & 0,24 \\
\hline Jun/ZES & 27,00 & 0,82 & 13,0 & 0,82 & 6,67 & 0,09 & 2,92 & 0,75 \\
\hline Jun/ZEM & 27,50 & 0,41 & 24,5 & 1,22 & 7,02 & 0,61 & 4,75 & 0,16 \\
\hline Jun/ZEI & 27,67 & 0,62 & 30,5 & 0,71 & 8,00 & 0,33 & 4,87 & 0,22 \\
\hline Ago/ZES & 25,17 & 0,62 & 11,5 & 4,02 & 7,31 & 0,15 & 3,77 & 0,57 \\
\hline Ago/ZEM & 25,67 & 0,47 & 20,0 & 1,63 & 7,72 & 0,12 & 3,78 & 0,04 \\
\hline Ago/ZEl & 26,83 & 0,62 & 28,0 & 1,08 & 8,15 & 0,19 & 4,7 & 0,37 \\
\hline
\end{tabular}

Revista Brasileira de Zoologia 25 (4): 647-661, December, 2008 
Tabela II. Famílias e espécies do estuário do Rio Formoso, com respectivo percentual numérico (\%PN); freqüência de ocorrência (\%FO); classificação quanto ao percentual numérico e freqüência de ocorrência (CNF); e categorias tróficas (CTR). (A-MF) Abundante e muito freqüente, (A-F) abundante e freqüente, (PA-MF) pouco abundante e muito freqüente, (PA-F) pouco abundante e freqüente, (PA-PF) pouco abundante e pouco freqüente, (C-I) carnívoro de primeira ordem, (C-II) carnívoro de segunda ordem, (C-III) carnívoro de terceira ordem, (P) planctívoro, (O) onívoro, (H) herbívoro, (ID) iliófago-detritívoro.

\begin{tabular}{|c|c|c|c|c|c|}
\hline Família & Espécie & $\% \mathrm{PN}$ & $\% \mathrm{FO}$ & CNF & CT \\
\hline Albulidae & Albula vulpes (Linnaeus, 1758) & 0,88 & 83,3 & PA-MF & C-I ${ }^{1}$ \\
\hline Muraenidae & Gymnothorax ocellatus Agassiz, 1831 & 0,04 & 33,3 & PA-PF & $\mathrm{C}-\|\|^{2}$ \\
\hline \multirow[t]{4}{*}{ Engraulidae } & Anchoa tricolor (Agassiz, 1829) & 0,16 & 16,6 & PA-PF & $\mathrm{P}^{3}$ \\
\hline & Anchovia clupeoides (Swainson, 1839) & 7,98 & 100,0 & A-MF & $\mathrm{P}^{4}$ \\
\hline & Cetengraulis edentulus (Cuvier, 1829) & 7,56 & 66,6 & A-F & $\mathrm{P}^{5}$ \\
\hline & Lycengraulis grossidens (Agassiz, 1829) & 1,02 & 50,0 & PA-F & $\mathrm{C}-1 \mathrm{II}^{6,7}$ \\
\hline \multirow[t]{3}{*}{ Clupeidae } & Opisthonema oglinum (Lesueur, 1818) & 0,04 & 16,6 & PA-PF & $\mathrm{P}^{7}$ \\
\hline & Harengula clupeola (Cuvier, 1829) & 6,41 & 100,0 & A-MF & $\mathrm{P}^{3,8}$ \\
\hline & Rhinosardinia amazonica (Steindachner, 1879) & 35,69 & 100,0 & A-MF & $P^{6}$ \\
\hline Synodontidae & Synodus foetens (Linnaeus, 1766) & 0,11 & 66,6 & PA-F & $\mathrm{C}-\mathrm{II}^{3,7}$ \\
\hline Batrachoididae & Thalassophryne nattereri Steindachner, 1876 & 0,22 & 83,3 & PA-MF & $\mathrm{C}-\mathrm{II}{ }^{9}$ \\
\hline \multirow[t]{2}{*}{ Antennariidae } & Antennarius striatus (Shaw \& Nodder, 1794) & 0,02 & 16,6 & PA-PF & $\mathrm{C}-11 \mathrm{II}^{17}$ \\
\hline & Histrio histrio (Linnaeus, 1758) & 0,02 & 16,6 & PA-PF & $\mathrm{C}-\mathrm{II}^{10}$ \\
\hline Ogcocephalidae & Ogcocephalus vespertilio (Linnaeus, 1758) & 0,02 & 16,6 & PA-PF & $C-\left.\right|^{11}$ \\
\hline Mugilidae & Mugil curema Valencienes, 1836 & 0,02 & 16,6 & PA-PF & $\mathrm{ID}^{7,12,13}$ \\
\hline Atherinopsidae & Atherinella brasiliensis (Quoy \& Gaimard, 1824) & 4,47 & 100,0 & A-MF & $\mathrm{O}^{3,12,14,15}$ \\
\hline Belonidae & Strongylura timucu (Walbaum, 1792) & 0,04 & 16,6 & PA-PF & $\mathrm{C}-1 \mathrm{II}{ }^{16}$ \\
\hline \multirow[t]{3}{*}{ Hemiramphidae } & Hemiramphus balao Lesueur, 1823 & 0,20 & 16,6 & PA-PF & C-II ${ }^{4,17}$ \\
\hline & Hyporhamphus roberti roberti (Valenciennes, 1846) & 0,02 & 16,6 & PA-PF & - \\
\hline & Hyporhamphus unifasciatus (Ranzani, 1842) & 0,13 & 50,0 & PA-F & $\mathrm{H}^{3,7}$ \\
\hline Poeciliidae & Poecilia vivipara Bloch \& Schneider, 1801 & 0,09 & 33,3 & PA-PF & $\mathrm{C}-\mathrm{I}^{18}$ \\
\hline Fistulariidae & Fistularia petimba Lacepède, 1803 & 0,05 & 33,3 & PA-PF & $\mathrm{C}-\mathrm{III}{ }^{19}$ \\
\hline Dactylopteridae & Dactylopterus volitans (Linnaeus, 1758) & 0,05 & 50,0 & PA-F & $C-1^{20}$ \\
\hline Scorpaenidae & Scorpaena isthmensis Meek \& Hildebrand, 1928 & 0,04 & 16,6 & PA-PF & $\mathrm{CII}^{22}$ \\
\hline Triglidae & Prionotus punctatus (Bloch, 1797) & 0,11 & 33,3 & PA-PF & C-II 20,21 \\
\hline \multirow[t]{3}{*}{ Centropomidae } & Centropomus undecimalis (Bloch, 1792) & 0,62 & 83,3 & PA-MF & $\mathrm{C}-1 \mathrm{I1}^{7,21}$ \\
\hline & Centropomus pectinatus Poey, 1860 & 4,51 & 100,0 & A-MF & $\mathrm{C}-1 \mathrm{II}^{22}$ \\
\hline & Centropomus parallelus Poey, 1860 & 0,47 & 83,3 & PA-MF & $\mathrm{C}-1 \mathrm{I}^{21,7}$ \\
\hline \multirow[t]{2}{*}{ Serranidae } & Epinephelus itajara (Lichtenstein, 1822) & 0,02 & 16,6 & PA-PF & $\mathrm{C}-\mathrm{II}^{21}$ \\
\hline & Rypticus saponaceus (Bloch \& Schneider, 1801) & 0,05 & 16,6 & PA-PF & $\mathrm{C}-1 \mathrm{II}^{16}$ \\
\hline \multirow[t]{6}{*}{ Carangidae } & Oligoplites palometa (Cuvier, 1833). & 0,13 & 50,0 & PA-F & $\mathrm{C}-1 \mathrm{II}^{7,23}$ \\
\hline & Oligoplites saurus (Bloch \& Schneider, 1801) & 0,15 & 33,3 & PA-PF & $\mathrm{C}-\mathrm{II}^{24}$ \\
\hline & Carangoides bartholomaei (Cuvier, 1833) & 0,02 & 16,6 & PA-PF & $\mathrm{C}-\mathrm{III}^{24}$ \\
\hline & Caranx crysos (Mitchill, 1815) & 0,22 & 33,3 & PA-PF & C-III \\
\hline & Caranx latus Agassiz, 1831 & 0,22 & 83,3 & PA-MF & $\mathrm{C}-1 \mathrm{II}^{7,24}$ \\
\hline & Selene vomer (Linnaeus, 1758) & 0,05 & 33,3 & PA-PF & C-III \\
\hline \multirow[t]{4}{*}{ Lutjanidae } & Lutjanus analis (Cuvier, 1828) & 0,11 & 33,3 & PA-PF & C-II ${ }^{17,24}$ \\
\hline & Lutjanus synagris (Linnaeus, 1758) & 0,60 & 100,0 & PA-MF & $\mathrm{C}-\mathrm{II}^{24}$ \\
\hline & Lutjanus jocu (Bloch \& Schneider, 1801) & 0,13 & 66,6 & PA-F & C-III,24 \\
\hline & Lutjanus griseus (Linnaeus, 1758) & 0,26 & 66,6 & PA-F & $\mathrm{C}-\mathrm{II}^{25}$ \\
\hline Gerreidae & Diapterus rhombeus (Cuvier 1829) & 8,51 & 100,0 & A-MF & $\mathrm{O}^{24,26}$ \\
\hline
\end{tabular}


Tabela II. Continuação.

\begin{tabular}{|c|c|c|c|c|c|}
\hline Família & Espécie & $\% \mathrm{PN}$ & $\%$ FO & CNF & CT \\
\hline \multirow[t]{6}{*}{ Gerreidae } & Diapterus rhombeus (Cuvier 1829) & 8,51 & 100,0 & A-MF & $\mathrm{O}^{24,26}$ \\
\hline & Eucinostomus argenteus (Baird \& Girard, 1855) & 1,74 & 100,0 & A-MF & $C-1^{20,24}$ \\
\hline & Eucinostomus gula (Cuvier, 1830) & 2,30 & 100,0 & A-MF & $C-17,24,27$ \\
\hline & Eucinostomus melanopterus (Bleeker, 1863) & 1,63 & 100,0 & A-MF & $\mathrm{O}^{28}$ \\
\hline & Eugerres brasilianus (Cuvier, 1830) & 3,76 & 100,0 & A-MF & $\mathrm{O}^{29,7}$ \\
\hline & Eucinostomus lefroyi (Goode, 1874) & 0,29 & 33,3 & PA-PF & $C-1^{24}$ \\
\hline \multirow[t]{2}{*}{ Haemulidae } & Haemulon parra (Desmarest, 1823) & 0,29 & 50,0 & PA-F & $C-1^{4}$ \\
\hline & Pomadasys corvinaeformis (Steindachner, 1868) & 0,04 & 16,6 & PA-PF & $\mathrm{O}^{30}$ \\
\hline Polynemidae & Polydactylus virginicus (Linnaeus, 1758) & 0,02 & 16,6 & PA-PF & $C-1^{31}$ \\
\hline \multirow[t]{2}{*}{ Sciaenidae } & Cynoscion leiarchus (Cuvier, 1830) & 0,24 & 16,6 & PA-PF & $\mathrm{C}-\mathrm{II}^{24,32}$ \\
\hline & Micropogonias furnieri (Desmarest, 1823) & 0,04 & 16,6 & PA-PF & $C-11^{24,32}$ \\
\hline Mullidae & Pseudupeneus maculatus (Bloch, 1793) & 0,11 & 16,6 & PA-PF & $C-1^{12,16}$ \\
\hline Pomacentridae & Stegastes fuscus (Cuvier, 1830) & 0,02 & 16,6 & PA-PF & $\mathrm{O}^{33}$ \\
\hline \multirow[t]{2}{*}{ Scaridae } & Sparisoma radians (Valenciennes, 1839) & 0,02 & 16,6 & PA-PF & $\mathrm{H}^{33}$ \\
\hline & Sparisoma amplum (Ranzani, 1842) & 0,24 & 66,6 & PA-F & $\mathrm{H}^{34}$ \\
\hline \multirow[t]{2}{*}{ Eleotridae } & Dormitator maculatus (Bloch, 1792) & 0,02 & 16,6 & PA-PF & $\mathrm{O}^{35}$ \\
\hline & Eleotris pisonis (Gmelin, 1789) & 0,02 & 16,6 & PA-PF & $\mathrm{C}-\mathrm{II}^{35}$ \\
\hline \multirow[t]{4}{*}{ Gobiidae } & Bathygobius soporator (Valenciennes, 1837) & 0,53 & 100,0 & PA-MF & $\mathrm{O}^{36}$ \\
\hline & Ctenogobius smaragdus (Valenciennes, 1837) & 0,07 & 50,0 & PA-F & - \\
\hline & Gobionellus oceanicus (Pallas, 1770) & 0,22 & 66,6 & PA-F & $\mathrm{ID}^{7}$ \\
\hline & Gobionellus stomatus Starks, 1913 & 0,51 & 66,6 & PA-F & - \\
\hline Ephippidae & Chaetodipterus faber (Broussonet, 1782) & 0,11 & 66,6 & PA-F & $\mathrm{O}^{33,7}$ \\
\hline Sphyraenidae & Sphyraena barracuda (Walbaum, 1792) & 0,11 & 66,6 & PA-F & $\mathrm{C}-1117,33$ \\
\hline \multirow[t]{2}{*}{ Scombridae } & $\begin{array}{l}\text { Scomberomorus brasiliensis Collete, Russo \& Zavala- } \\
\text { Camin, } 1978\end{array}$ & 0,02 & 16,6 & PA-PF & $\mathrm{C}-1 \mathrm{II}^{7}$ \\
\hline & Scomberomorus cavalla (Cuvier, 1829) & 0,04 & 16,6 & PA-PF & $\mathrm{C}-\mathrm{III}{ }^{16}$ \\
\hline \multirow[t]{4}{*}{ Paralichthyidae } & Citharichthys arenaceus Evermann \& Marsh, 1902 & 0,20 & 86,6 & PA-MF & $\mathrm{C}-111^{37}$ \\
\hline & Citharichthys spilopterus Günther, 1862 & 2,78 & 100,0 & PA-MF & $\mathrm{C}-\mathrm{II}^{7,37,38}$ \\
\hline & Etropus crossotus Jordan \& Gilbert, 1882 & 0,02 & 16,6 & PA-PF & $C-1^{37,} 39,40,41$ \\
\hline & Paralichthys brasiliensis (Ranzani, 1840) & 0,02 & 16,6 & PA-PF & - \\
\hline \multirow[t]{2}{*}{ Achiridae } & Achirus declivis Chabanaud, 1940 & 0,11 & 50,0 & PA-F & - \\
\hline & Achirus lineatus (Linnaeus, 1758) & 0,13 & 66,6 & PA-F & $C-I^{7,37,42}$ \\
\hline \multirow[t]{2}{*}{ Cynoglossidae } & Symphurus plagusia (Bloch \& Schneider, 1801) & 0,07 & 66,6 & PA-F & $C-17$ \\
\hline & Symphurus tessellatus (Quoy \& Gaimard, 1824) & 0,26 & 86,6 & PA-MF & $C-1^{38}$ \\
\hline Ostraciidae & Lactophrys trigonus (Linnaeus, 1758) & 0,04 & 16,6 & PA-PF & $C-1^{16}$ \\
\hline \multirow[t]{3}{*}{ Tetraodontidae } & Sphoeroides greeleyi Gilbert, 1900 & 1,72 & 100,0 & A-MF & $C-1^{40}$ \\
\hline & Sphoeroides testudineus (Linnaeus, 1758) & 0,60 & 100,0 & PA-MF & $\mathrm{O}^{41,42}$ \\
\hline & Sphoeroides spengleri (Bloch, 1785) & 0,18 & 50,0 & PA-F & $C-1^{40}$ \\
\hline Diodontidae & Cyclichthys antillarum (Jordan \& Rutter, 1897) & 0,02 & 16,6 & PA-PF & - \\
\hline
\end{tabular}

(1) Lopes (1999), (2) Santos \& Castro (2003), (3) Figueiredo \& Menezes (1978), (4) Sierra et al. (1994), (5) Gay et al. (2002), (6) Whitehead et al. (1988), (7) Vasconcelos-Filho et al. (2003), (8) Randall (2004), (9) Chacas et al. (2004), (10) Rooker et al. (2006), (11) Gibran \& Castro (1999), (12) Sazima (1986), (13) Franco \& Bashirullah (1992), (14) Furtado (1969), (15) Bemvenuti (1990), (16) Randall (2004), (17) Randall (1967), (18) Froese \& Pauly (2007), (19) Bowman et al. (2000), (20) Soares et al. (1993), (21) Figuelredo \& Menezes (1980), (22) Cervigón (1989) (23) Sazima (1983), (24) Menezes \& Figueiredo (1980), (25) Franks \& Vanderkooy (2000), (26) Chaves \& Otto (1998), (27) Zahorcsak et al. (2000), (28) Chaves \& Robert (2001), (29) Chaves \& Corrêta (2000), (30) Austin \& Austin (1971), (31) Chaves \& Umbria (2003), (32) Menezes \& Figueiredo (1985), (33) Cervigón (1993), (34) Ferreira \& Gonçalves (2006), (35) Teixeira (1994), (36) Chaves \& Serenato (1998), (37) Guedes et al. (2004), (38) Castillo-Rivera et al. (2000), (39) Figueiredo \& Menezes (2000), (40) Lunardon-Branco \& Branco (2003), (41) Almeida et al. (1997), (42) VAsConcelos-Filho et al. (1998). 

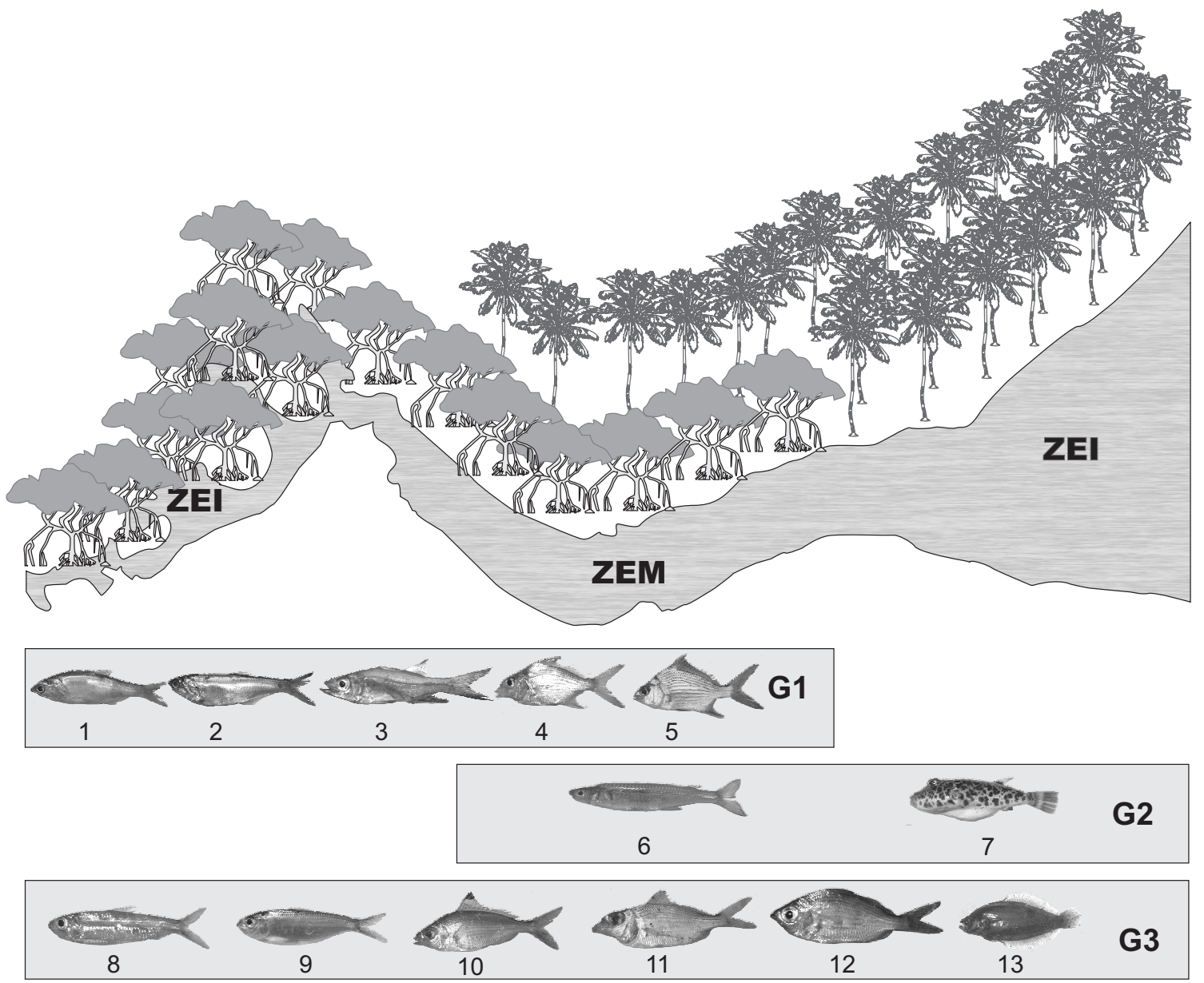

Figura 4. Distribuição espacial das espécies abundantes e muito freqüentes e abundantes e freqüentes em águas rasas nas zonas estuarinas do Rio Formoso: (1) C. edentulous, (2) A. clupeoides, (3) C. pectinatus, (4) E. brasilianus, (5) D. rhombeus, (6) A. brasiliensis, (7) S. greeleyi, (8) H. clupeola, (9) R. amazonica, (10) E. melanopterus,; (11) E. gula, (12) E. argenteus, (13). E. brasilianus. (G1) grupo 1, (G2) grupo 2, (G3) grupo 3.

Através da ANOVA foi possível verificar que houve diferenças estatisticamente significativas na diversidade entre as zonas estuarinas superior e inferior $\left(\mathrm{F}_{(2 ; 10)}=6,3528 ; \mathrm{p}=0,0166\right)$, sendo maior na zona estuarina superior (Tab. IV), entretanto essas diferenças não foram detectadas entre os meses de amostragem $\left(\mathrm{F}_{(5 ; 10)}=1,5070 ; \mathrm{p}=0,2713\right)$.

A maioria (71\%) das espécies que constituem a ictiofauna do Rio Formoso enquadrou-se como carnívora. As diferentes categorias de hábitos laimentares são descritas na figura 5.

As categorias tróficas da ictiofauna de águas rasas do Rio Formoso não diferiram estatisticamente entre as zonas estuarinas ( $F=0,6106 ; p=0,5583)$, nem entre os meses dos períodos seco e chuvoso $[\mathrm{t}=-0,0380 ; \mathrm{p}$ (bilateral) $=0,9703]$. Entretanto, as espécies carnívoras de segunda ordem, seguidas pelas carnívoras de primeira ordem, predominaram tanto nas zonas estuarinas superior, média e inferior, como nos períodos seco e chuvoso (Tab. V).
Na zona estuarina superior e no período seco os valores de Fator de Contribuição Trófica para detritos superaram o da produção primária, situação inversa à registrada na zona estuarina média e no período chuvoso (Fig. 6). Na zona estuarina inferior, os valores do Fator foram semelhantes para detritos e produção primária.

\section{DISCUSSÃO}

Os peixes de estuários tropicais estão sujeitos a uma gama de interações de fatores físico-químicos e biológicos que determinam seus padrões de ocorrência, distribuição e movimento (BLAber 2000). No estuário do Rio Formoso os valores de temperatura, salinidade, $\mathrm{pH}$ e oxigênio dissolvido foram mais elevados na zona estuarina inferior, e durante o período seco. Em regiões estuarinas de Pernambuco, as variações sazonais de temperatura e salinidade das águas são bem evidenciadas, sendo registrados os valores mais elevados nos meses do período seco 
Tabela III. Número de indivíduos das espécies abundantes e muito freqüentes (A-MF) e abundantes e freqüentes (A-F) de águas rasas no estuário do Rio Formoso, nos meses de período seco e chuvoso. (out) Outubro, (dez): dezembro, (fev): fevereiro, (abr): abril, (jun) junho, (ago) agosto, (PS) período seco, (PC) período chuvoso.

\begin{tabular}{lrrrrrrrr}
\hline \multicolumn{1}{c}{ Espécie } & Out & Dez & Fev & Total PS & Abr & Jun & Ago & Total PC \\
\hline Cetengraulis edentulus & 0 & 97 & 0 & 97 & 112 & 80 & 125 & 317 \\
Anchovia clupeoides & 105 & 57 & 90 & 252 & 165 & 4 & 16 & 185 \\
Rhinosardinia amazonica & 217 & 235 & 77 & 529 & 757 & 520 & 148 & 1425 \\
Harengula clupeola & 29 & 228 & 32 & 289 & 6 & 30 & 26 & 62 \\
Atherinella brasiliensis & 10 & 79 & 35 & 124 & 68 & 43 & 10 & 121 \\
Centropomus pectinatus & 227 & 1 & 5 & 233 & 2 & 2 & 10 & 14 \\
Eugerres brasilianus & 39 & 11 & 124 & 174 & 14 & 7 & 11 & 32 \\
Diapterus rhombeus & 83 & 85 & 79 & 247 & 22 & 85 & 112 & 219 \\
Eucinostomus argenteus & 19 & 7 & 11 & 37 & 21 & 27 & 10 & 58 \\
Eucinostomus gula & 26 & 6 & 56 & 88 & 20 & 15 & 3 & 38 \\
Eucinostomus melanopterus & 6 & 20 & 10 & 36 & 14 & 22 & 17 & 53 \\
Citharichthys spilopterus & 29 & 12 & 11 & 52 & 19 & 51 & 30 & 100 \\
Sphoeroides greeleyi & 21 & 16 & 14 & 51 & 19 & 12 & 12 \\
\hline
\end{tabular}

Tabela IV. Resultados do teste de Tukey para a diversidade entre as zonas estuarinas superior (ZES), média (ZEM) e inferior (ZEI) do Rio Formoso.

\begin{tabular}{cl}
\hline Zonas estuarinas & Média \\
\hline ZES & $2,6613 \mathrm{a}$ \\
ZEM & $1,7745 \mathrm{ab}$ \\
ZEI & $1,5263 \mathrm{~b}$ \\
\hline
\end{tabular}

Tabela V. Número de espécies da ictiofauna de águas rasas, pertencentes a cada categoria trófica nas zonas estuarinas superior (ZES), média (ZEM) e inferior (ZEI) do Rio Formoso; e nos períodos seco (PS) e chuvoso (PC).

\begin{tabular}{lrrrrr}
\hline \multicolumn{1}{c}{ Categorias tróficas } & ZES & ZEM & ZEI & PS & PC \\
\hline Carnívoro de Primeira ordem & 8 & 10 & 7 & 16 & 13 \\
Carnívoro de segunda ordem & 15 & 19 & 10 & 20 & 20 \\
Carnívoro de terceira ordem & 2 & 3 & 3 & 4 & 4 \\
Herbívoro & 0 & 2 & 2 & 3 & 2 \\
Iliófago-detritívoro & 1 & 1 & 0 & 1 & 1 \\
Onívoro & 8 & 7 & 3 & 7 & 10 \\
Planctívoro & 5 & 6 & 2 & 4 & 6 \\
\hline
\end{tabular}

(janeiro a março) (MACÊDo et al. 2000, 2004), conforme foi verificado no presente estudo.

Os valores de $\mathrm{pH}$ e oxigênio dissolvido são influenciados pelos ciclos de marés e taxas de fotossíntese e/ou respiração (MACÊDo et al. 2000), entretanto a capacidade de neutralização existente no ecossistema aquático devido ao efeito buffer (tampão) impede que ocorram amplas variações do $\mathrm{pH}$, e os valores máximos são obtidos nas áreas de maior influência salina (MACÊDo et al. 2000). No estuário do Rio Formoso os valores mais elevados de $\mathrm{pH}$ foram registrados na zona estuarina inferior, que possui salinidade mais elevada. Nesta zona o teor de oxigênio dissolvido também foi maior, concordando com MACÊDo et al. (2000), que afirmam que os valores mais elevados desse gás são registrados em áreas mais distantes das desembocaduras dos rios, como é o caso da zona estuarina inferior. No estuário do Rio Botafogo (Pernambuco) as concentrações de oxigênio dissolvido mostraram-se irregulares, onde níveis críticos foram observados principalmente nas estações à montante da desembocadura (MACÊDo et al. 2004). Fato semelhante ocorreu no presente estudo, onde os valores mais baixos de oxigênio dissolvido foram registrados nas zonas mais internas (superior e média) do Rio Formoso. No Canal de Santa Cruz, Itamaracá, Pernambuco, os valores de oxigênio dissolvido variaram entre 2,02 e 5,51 e ml.1 $1^{-1}$ (MACÊDo et al. 2000), enquanto no presente estudo o mínimo e o máximo foi 2,92 e 5,91, respectivamente. Esses resultados sugerem que a poluição orgânica gerada pelo lançamento de esgotos domésticos na porção desse estuário próxima à cidade de Rio Formoso (Melo et al. 2002) não chega a atingir as áreas estudadas no presente trabalho, fato corroborado por esses mesmos autores, que comentam que a poluição nesse rio diminui gradativamente da cidade até o estuário. Os valores percentuais de silte e argila variaram entre as zonas estuarinas, sendo maior na zona estuarina superior, fato corroborado através do estudo de LiRA et al. (1979) que verificaram diferentes tipos de sedimento entre as zonas estuarinas superior, média e inferior do Rio Formoso.

É difícil comparar a riqueza de espécies da ictiofauna entre diferentes estuários devido à heterogeneidade de hábitats, diferenças físico-químicas no ambiente, assim como diferenças 


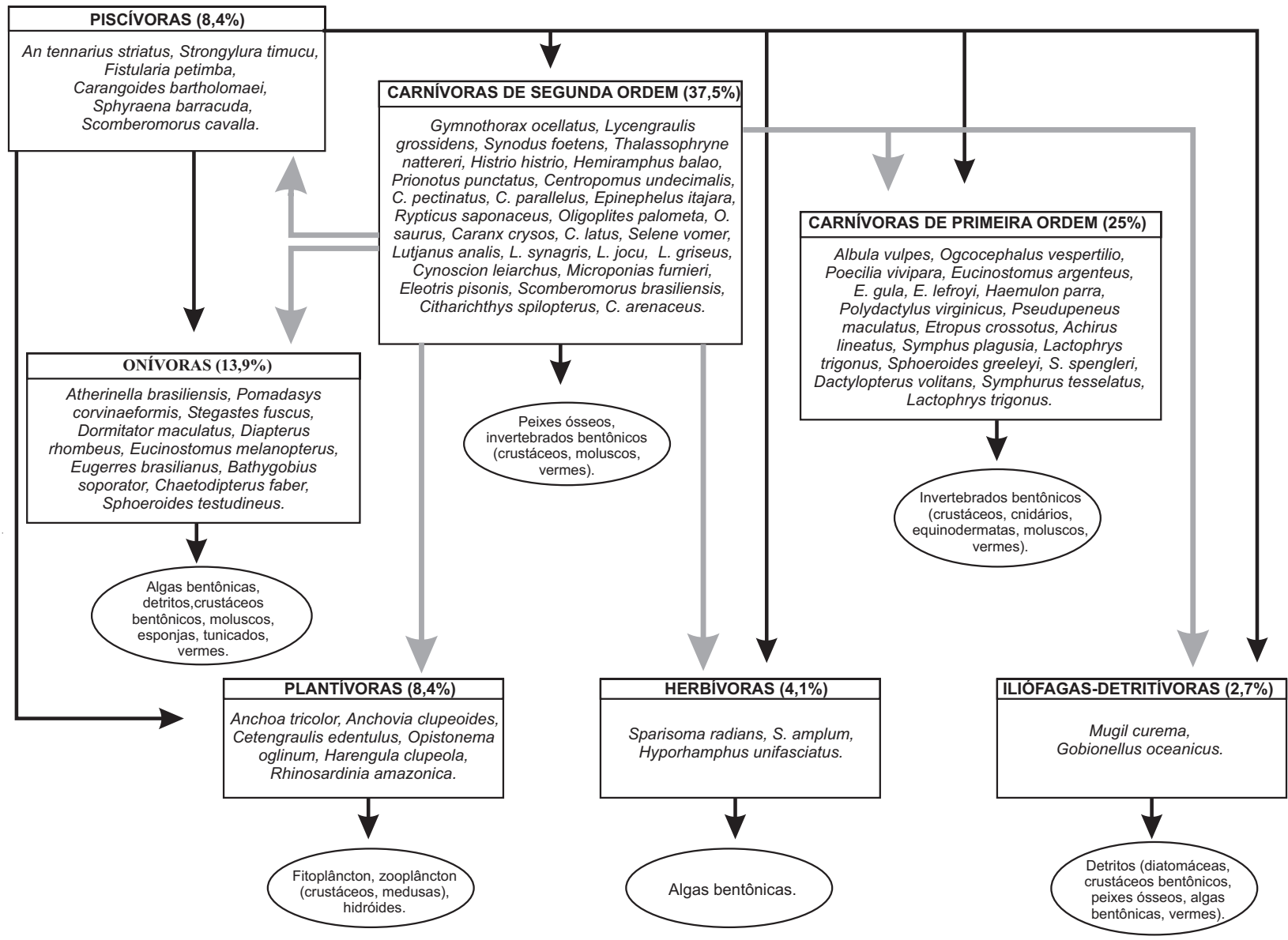

Figura 5. Estrutura trófica da comunidade de peixes estuarinos do Rio Formoso.
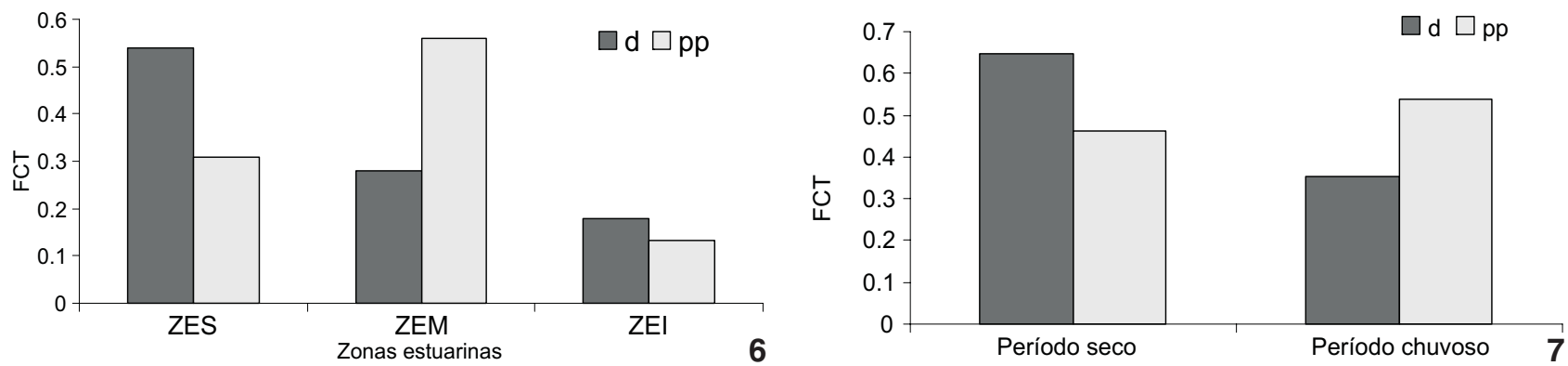

Figuras 6-7. Fator de Contribuição Trófica nas zonas estuarinas superior (ZES), média (ZEM) e inferior (ZEI) do Rio Formoso (7), e entre os períodos seco e chuvoso (8).

no esforço de captura (ARAújo et al. 1998). Os índices de diversidade numérica e de peso são influenciados por diversos fatores, como época do ano, local de amostragem e seletividade dos petrechos de pesca (Villarroel 1994). Apesar dessas restrições, a ictiofauna estuarina de águas rasas do Rio Formoso possui elevado número de espécies (78) quando comparado com outros estuários do Atlântico oeste tropical: Rio Caeté, Pará (BARLETta et al. 2003), Rio Tibiri e Rio Paciência, Maranhão (BATISTA \& Rego 1996, CASTRO 2001), Rio Jaguaribe, Ceará (Alves \& SoAres-Filho 1996) e estuário de Cacha Pregos, Bahia (Lopes et al. 1998), onde foi realizado um maior número de amostragens, utilizando-se petrechos de pesca com maiores dimensões. 
No presente estudo, Carangidae e Gerreidae apresentaram maior riqueza específica, assim como na gamboa de Sucuriú (Spach et al. 2003). Nos estuários do Rio Caeté (Barletta et al. 2003), Rio Paciência (CASTro 2001), Rio Jaguaribe (Alves \& SoAREs-FilHo 1996) e Cacha Pregos (Lopes et al. 1998), essas famílias estiveram entre as de maior riqueza específica. Carangidae está entre as famílias com maior riqueza em $65 \%$ dos estuários analisados no presente estudo, enquanto Gerreidae em 29\% desses estuários. Em baías e lagoas do Estado do Rio de Janeiro a grande ocorrência de Gerreidae em estágio juvenil está associada à margem continental, ambiente ideal para o seu desenvolvimento nos primeiros estágios de vida (ARAújo et al. 1997).

Os indivíduos provenientes das margens dos estuários são geralmente jovens de espécies de importância econômica pertencentes a Clupeidae, Mugilidae, Centropomidae, Carangidae, Lutjanidae, Gerreidae, Haemulidade, e Sciaenidae (GuEdes et al. 2005). As zonas rasas dos estuários também abrigam maior contingente de peixes tipicamente estuarinos (DAY et al. 1989). No presente estudo, todos os exemplares das famílias citadas encontravam-se em ecofase jovem. Uma das características da população de peixes em ambientes de águas salobras costeiras é a abundância de juvenis (SpACH et al. 2004). O elevado número de espécies, principalmente em ecofase jovem, evidencia a importância das águas rasas do estuário do Rio Formoso, como criadouro natural e abrigo para várias espécies de peixes de importância ecológica e econômica, incluindo àquelas de origem recifal, que representaram 51,3\% do número total de espécies. Assim, torna-se necessária uma maior fiscalização pelos órgãos públicos competentes, a fim de impedir o uso de redes com aberturas de malha pequena, para evitar a captura de espécies muito jovens. Ainda, devem ser implantados planos de manejo para a conservação não apenas da ictiofauna que utiliza esse estuário como berçário, mas de toda a biota do Rio Formoso.

Nos ambientes estuarinos, os manguezais fornecem refúgio natural para indivíduos jovens devido à proteção fornecida pela estrutura radicular de suas árvores. A maior parte dos peixes capturados nas áreas litorâneas tropicais desfruta desta proteção durante sua fase jovem e na época da postura e, assim, dependem intimamente da integridade desse ecossistema (LACERDA 1984).

Exceto por $A$. brasiliensis (Quoy \& Gaimard, 1824) e $R$. amazonica, as espécies de maior abundância nas águas rasas do Rio Formoso destacaram-se também como abundantes e freqüentes no Canal de Santa Cruz, Pernambuco (Eskinazi 1972). Atherinella brasiliensis também foi considerada uma das espécies mais representativas no estuário do Rio Jaguaribe, Ceará (Freitas et al. 2006), nas Baías de Laranjeiras e de Paranaguá, Paraná (FAlCão et al. 2006), na Gamboa do Sucuriú, Paraná (SPACH et al. 2003) e na Laguna do Imaruí, Santa Catarina (MonteiroNeto et al. 1990). Embora R. amazonica tenha sido a mais abundante e freqüente na presente pesquisa, o seu primeiro registro para Pernambuco data de 2005 (Guedes et al. 2005). É provável que até então esta espécie tenha sido confundida com $P$. platana
Regan, 1917 cuja distribuição não atinge o nordeste do Brasil (José Lima de Figueiredo, Museu de Zoologia, Universidade de São Paulo. com. pess.).

Os padrões espaciais da comunidade de peixes estão associados principalmente às diferenças nos hábitats, às variações sazonais (SAUL \& CunNingham 1995) e ao tipo de substrato (Lowe-McConnell 1999, Amezcua \& NASH 2001). No presente estudo a maioria dos peixes demersais foi coletada nas zonas mais internas do estuário (superior e média), provavelmente devido à maior complexibilidade morfológica desta zona, e ao incremento nos teores de lama (silte e argila). Nas águas barrentas, com elevadas cargas de partículas em suspensão, como geralmente ocorre na região interna dos estuários, registram-se comunidades predominantemente demersais, que têm como principal fonte de alimento os organismos bentônicos (CAMARGO \& ISAAC 2003). Os teores de silte e argila no substrato são diretamente proporcionais à matéria orgânica (PAIVA et al. 2005), indicando que nas zonas mais internas do Rio Formoso há um maior incremento desse componente. Vários peixes demersais estão associados a um tipo particular de sedimento (GIBSON \& Rовв 1992), onde obtêm o alimento constituído principalmente pelos invertebrados bentônicos (Almeida et al. 1997, Labropoulou \& Papadopoulou 1999).

Estudos como os de Villarroel (1994) e Barletta et al. (2003) indicam que as variações temporais pouco influenciam na estrutura da comunidade dos peixes estuarinos. Na Baía de Sepetiba (RJ), os padrões sazonais de abundância da ictiofauna foram pouco evidentes, entretanto o maior número de indivíduos foi comparativamente maior de fevereiro a junho, meses que coincidiram com as maiores temperaturas e transparência das águas (Araújo et al. 1998). No presente estudo, apesar de não existirem diferenças significativas na diversidade da ictiofauna entre os períodos seco e chuvoso, houve incremento de indivíduos no período chuvoso, correspondendo a $54,46 \%$ do número de indivíduos coletados. Diferentemente, na gamboa do Sucuriú (PR), a variação na estrutura da comunidade de peixes foi definida principalmente pelo padrão de ocorrência de espécies associadas preferencialmente a águas mais salinas, ocorrendo uma relação direta entre a variabilidade amostral e o aumento da transparência e salinidade da água, e inversa com o incremento da pluviosidade (SpAch et al. 2003). A ausência de um padrão sazonal na ictiofauna pode ser justificada pela dominância numérica de algumas espécies que apresentam picos de abundância de forma aleatória (GodEFroid et al. 2004). No estuário do Rio Formoso, a dominância de R. amazonica e D. rhombeus (Cuvier, 1829) em todos os meses de amostragem pode ter contribuído para a ausência de diferenças significativas na distribuição temporal da ictiofauna. Isso pode ser verificado quando o número de indivíduos dessas espécies é excluído da tabela, ficando a maior abundância de peixes no período seco.

No presente estudo, H. clupeola, E. brasilianus e $C$. pectinatus destacaram-se como mais abundantes no período seco, enquanto $R$. amazonica, C. edentulus e C. spilopterus, no 
período chuvoso. Na Gamboa do Sucuriú, H. clupeola e C. edentulus foram mais abundantes entre fevereiro e abril, meses que coincidem com as maiores precipitações pluviométricas (SPACH et al. 2003), enquanto na Gamboa do Baguaçu, H. clupeola foi dominante entre os meses de março a julho, com menores índices pluviométricos (SpACH et al. 2004). Nota-se, portanto, que para essas espécies não há um padrão de distribuição temporal definido. Dependendo do local de amostragem, podem predominar em épocas seca ou chuvosa. Nas águas rasas do estuário do Rio Formoso a maior abundância de C. pectinatus no período seco deve-se ao fato de, em outubro, a espécie ter sido coletada em uma área próxima a "tocos" de árvores de mangue. Nesses locais ocorre grande abundância de peixes em ecofase jovem, e provavelmente estavam sendo utilizados por C. pectinatus para se protegerem de ataques de predadores. De acordo com relato de pescadores locais, esses "tocos" funcionam como atrativos para os peixes jovens que lá se abrigam, assim como os rizóforos de Rhizophora mangle Linnaeus, que são refúgios para muitas outras espécies de peixes jovens (MuMBY 2006).

A ictiofauna de estuários e lagunas é caracterizada por uma forte proporção de espécies predadoras (em torno de 80\%), porém a maioria não é especializada, consumindo vários grupos de invertebrados e vertebrados (BLABER 2000). Essa afirmação é válida também para a comunidade de peixes de águas rasas do estuário do Rio Formoso, onde 71\% das espécies apresentaram hábito alimentar carnívoro. A dominância de espécies com dieta generalizada com forte tendência à carnivoria (especialmente invertebrados) sobre outras categorias concorda com o encontrado em outros estuários. Em um estuário de Borneo, Indonésia, 69\% das espécies foram classificadas como carnívoras (Blaber 2000); e na Baía de Guaratuba, Paraná, este hábito foi evidenciado em 64,9\% das espécies (CHAves \& Bouchereau 2004). Quanto à classificação das categorias de carnivoria, a maior percentagem $(37,5 \%)$ de espécies no estuário do Rio Formoso foi de carnívoros de segunda ordem, ou seja, consumidores de invertebrados bentônicos e peixes. Resultados semelhantes foram verificados na Baía de Guaratuba, com 38,6\% (Chaves \& Bouchereau 2004). Em Manche-à-Eau, Antilhas Francesas, essa categoria trófica foi verificada em 20,1\% das espécies de peixes (CABERTy et al. 2004). Já em Borneo não foram encontradas espécies carnívoras de segunda ordem, sendo a maior proporção (50\%) de carnívoros de primeira ordem (Blaber 2000). Muitos peixes que são piscívoros quando adultos predam invertebrados bentônicos quando juvenis. É o caso de alguns representantes de Carangidae, entretanto $C$. bartolomaei (Cuvier, 1833) é piscívoro mesmo em estágio juvenil (Froese \& Pauly 2007). No presente estudo, a categoria trófica carnívoros de primeira ordem foi uma das mais representativas, com 25,0\% das espécies. De fato, para os peixes em estágio larval e várias espécies de Gobiidae, a meiofauna constitui o principal item alimentar, assim como os invertebrados bentônicos para os peixes em estágio juvenil de muitas espéci- es de Haemulidade, Sparidae e Gerreidae (Blaber 2000). Os peixes planctívoros estão representados principalmente por Clupeidae e Engraulidae, que variam em abundância de acordo com a biomassa e composição do plâncton, assim como com as características físicas e localização dos estuários (BLABER 2000). No estuário do Rio Formoso, além dessas famílias, os planctívoros estiveram representados por Hemiramphidae. De acordo com Figueiredo \& Menezes (1978) os exemplares pequenos e de tamanho médio de $H$. unifasciatus (Ranzani, 1842) consomem principalmente algas.

O Fator de Contribuição Trófica (FCT) é usado para caracterizar a parcela de participação dos elementos que constituem a base da teia trófica estuarina: detritos e produção primária (CHAves \& Bouchereau 2004). Entre as zonas estuarinas, o FCT para os detritos foi mais elevado na zona estuarina superior; enquanto para a produção primária foi maior na zona estuarina média. No primeiro caso o fato é justificado pela maior biomassa das espécies de águas rasas do Rio Formoso estar mais diretamente relacionadas com os detritos, como iliófagosdetritívoros, carnívoros de primeira ordem e onívoros, na zona estuarina superior. Quanto à produção primária, SILVA et al . (2004) verificaram que a zona estuarina média apresentou maior número de espécies de fitoplâncton. Esse fato pode estar associado ao maior FCT para a produção primária, uma vez que houve uma grande participação em biomassa de espécies planctívoras nessa zona.

Normalmente, a maior proporção de detritos ocorre em ambientes com elevada quantidade de matéria orgânica (QASIM \& Sankaranarayanan 1972). Os detritos são ingeridos como alimento por uma grande variedade de animais estuarinos, constituindo um elo entre a produção orgânica e a nutrição animal, o que auxilia a eficiência da transferência de energia entre os níveis tróficos (QASIM \& SANKARANARAYANAN 1972). Sazonalmente, nas águas rasas do estuário do Rio Formoso, o FCT para os detritos foi maior no período seco, o que diverge do encontrado por Chaves \& Bouchereau (2004) para a Baía de Guaratuba, onde o valor foi mais elevado na estação chuvosa. Em um estuário tropical da Índia QASIM \& SANKARANARAYANAN (1972) verificaram que a quantidade de detritos foi baixa nos períodos de monção e pós-monção (julho a dezembro), quando a turbidez no estuário foi máxima e coincidiu com as elevadas precipitações pluviométricas. Os autores explicam que esse fato se deve a estratificação desenvolvida no estuário nos meses de junho a dezembro, como resultado da descontinuidade nos perfis verticais de temperatura e salinidade. Isso, provavelmente, reduziu substancialmente a taxa de assentamento dos detritos (QASIM \& Sankaranarayanan 1972). Apesar de não ter sido verificado o perfil vertical da temperatura e salinidade no estuário do Rio Formoso, é possível que a baixa contribuição do FCT para os detritos no período chuvoso possa estar relacionada com essas variáveis hidrológicas. Isso possivelmente reduziu o número de espécies detritívoras no período chuvoso, diminuído o FCT para esse período. 


\section{AGRADECIMENTOS}

Sigrid Neumann-Leitão e Alessandra C. da Silva orientaram a aplicação das análises estatísticas; Caroline Vieira Feitosa ofereceu sugestões para a primeira versão deste manuscrito; a Fundação de Amparo à Ciência e Tecnologia do Estado de Pernambuco, a Coordenação de Aperfeiçoamento de Pessoal de Nível Superior e ao Conselho Nacional de Pesquisa concederam bolsa aos autores; e o Instituto Nacional de Meteorologia forneceu dados pluviométricos.

\section{LITERATURA CITADA}

Almeida, Z.S.; V. FonsÊCA-Genevois \& A.L. Vasconcelos-Filho. 1997. Alimentação de Achirus lineatus (Teleostei, Pleuronectiforme: Achiridae) em Itapissuma - PE. Boletim do Laboratório de Hidrobiologia 10: 79-95.

Alves, M.I.M. \& A.A. Soares-Filho. 1996. Peixes do estuário do rio Jaguaribe (Ceará- Brasil): aspectos fisioecológicos. Ciência Agronômica 27 (1/2): 5-16.

Amezcua, F. \& R.D.M. Nash. 2001. Distribution of the order Pleuronectiformes in relation to the sediment type in the North Irish Sea. Journal of Sea Research 45: 293-301.

Araújo, F.G.; A. Cruz-Filho; M.C. Azevêdo; A.C.A. Santos \& L.A.M. FERNANDES. 1997. Estrutura da comunidade de peixes jovens da margem continental da Baía de Sepetiba, RJ. Acta Biologica Leopoldensia 19 (1): 61-83.

Araújo, F.G.; A.G. Cruz-Filho; M.C.C. Azevêdo \& A.C.A. Santos. 1998. Estrutura da comunidade de peixes demersais da Baía de Sepetiba, RJ. Revista Brasileira de Biologia 58 (3): 417-430.

Araújo, M.E.; J.M.C. Teixeira \&. A.M.E. Oliveira. 2004. Peixes estuarinos marinhos do nordeste brasileiro. Guia Ilustrado. Recife, Editora Universitária, UFPE e EFC, 260p.

Austin, H. \& S. Austin. 1971. The feeding habits of some juvenile marine fishes from the mangroves in western Puerto Rico. Caribbean Journal Science 11 (3-4): 171-178.

Barletta, M.; A. Barletta-Bergan; U. Saint-Paul \& G. Hubold. 2003. Seasonal changes in density, biomass, and diversity of estuarine fishes in tidal mangrove creeks of the lower Caeté Estuary (northern Brazilian coast, east Amazon). Marine Ecology Progress Series 256: 217-228.

Batista, V.S. \& F.N. Rego. 1996. Análise de associações de peixes em igarapés do estuário do rio Tibiri, Maranhão. Revista Brasileira de Biologia 56 (1): 163-176.

Bowman, R.E.; C.E. Stillwell; W.L. Michaels \& M.D. Grosslein. 2000. Food of northwest Atlantic fishes and two common species of squid. NOAA Technical Memorandum. NMFSNE 155: 1-138.

Bemvenuti, M.A. 1990. Hábitos alimentares de peixes-rei (Atherinidae) na região estuarina da Lagoa dos Patos, RS, Brasil. Atlântica 12 (1): 79-102.

BlabER, S.J.M. 2000. Tropical estuarine fishes: ecology, exploitation and conservation. Queensland, Blackwell Science, $372 \mathrm{p}$.

Revista Brasileira de Zoologia 25 (4): 647-661, December, 2008
Bouchon-Navaro, Y.; C. Bouchon \& M. Louis. 1992. L'ichtyofaune des herbiers de phanérogames marines de la baie de Fortde-France (Martinique, Antilles Françcaises). Cybium 16 (4): 307-330.

Caberty, S.; J. Bouchereau \& P.T. Chaves. 2004. Organisation et fonctionnement trophiques de l'assemblage ichtyque d'um écosystème lagunaire à mangrove antillais au moyen de l'indice trophique de contribution. Cahier de Biologie Marine 45: 243-254.

Camargo, M. \& V.J. IsaAc. 2003. Ictiofauna estuarina, p. 105-142. In: M.E.B. Fernandes (Ed.). Os manguezais da costa Norte brasileira. São Luís, Fundação Rio Bacanga, vol. 1, 257p.

Castillo-Rivera, M.; A. Kobelkowsky \& A.M. Chávez. 2000. Feeding biology of the Citharichthys spilopterus (Bothidae) in a tropical estuary of México. Journal Applied Ichthyology 16: 73-78.

Castro, A.C.L. 2001. Diversidade da assembléia de peixes em igarapés do estuário do rio Paciência (MA - BRASIL). Atlântica 23: 61-72.

Cervigón, F. 1989. Los peces marinos de Venezuela. Caracas, Editorial Arte, 425p.

Cervigón, F. 1991. Los peces marinos de Venezuela. Caracas, Fundación Científica Los Roques, $2^{\text {nd }}$ ed., vol. 1, 425p.

Cervigón, F. 1993. Los peces marinos de Venezuela. Caracas, Fundación Científica Los Roques, vol. 2, 497p.

Cervigón, F. 1994. Los peces marinos de Venezuela. Caracas, Fundación Científica Los Roques, vol. 3, 295p.

Cervigón, F. 1996. Los peces marinos de Venezuela. Caracas, Fundación Científica Los Roques, vol. 4, $2^{\mathrm{a}}$ ed., 295p.

Chagas, R.B.; P.R.D. Lopes \& J.T. Oliveira-Silva. 2004. Nota sobre alimentação de Thalassophryne sp. (Actinopterygii: Batrachoididade) na praia de Cabuçu (Saubara, Baía de Todos os Santos, Bahia). Revista de Biociências 10 (4): 231234.

Chaves, P.T. \& J.L. Bouchereau. 2004. Trophic organization and functioning of fish populations in the Bay of Guaratuba, Brazil, on the basis of a trophic contribution factor. Acta Adriatica 45 (1): 83-94.

Chaves, P.T.C. \& C.E. CorrêA. 2000. Temporary use of coastal ecosystem by the fish, Pomadasys corvinaeformis (Perciformes: Haemulidae), at Guaratuba Bay, Brazil. Revista Brasileira de Oceanografia 48 (1): 1-7.

Chaves, P.T.C. \& G. Otтo. 1998. Aspectos biológicos de Diapterus rhombeus (Cuvier) (Teleostei, Gerreidae) na Baía de Guaratuba, Paraná, Brasil. Revista Brasileira de Zoologia 15 (2): 289-295.

Chaves, P.T. \& M.C. Robert. 2001. Nota complementar sobre os hábitos de Gerres melanopterus (Teleostei: Gerreidae) na Baía de Guaratuba (PR, Brasil). Revista Brasileira de Zoologia 18 (1): 255-259.

Chaves, P.T.C. \& A. Serenato. 1998. Diversidade de dietas na assembléia de linguados (Teleostei, Pleuronectiformes) do manguezal da Baía de Guaratuba, Paraná, Brasil. Revista 
Brasileira de Oceanografia 46 (1): 61-68.

Chaves, P.T.C. \& S. Umbria. 2003. Mudanças na dieta de peixes que se deslocam entre dois sistemas costeiros, estuário e Plataforma Continental. Brazilian Archives of Biology and Technology 46 (1): 41-46.

Condere. 1992. Monografias Municipais: Rio Formoso. Recife, Condepe, vol. 2, 173p.

CPRH . 1991. Alternativas de uso e proteção dos manguezais do Nordeste. Recife, Companhia Pernambucana do Meio Ambiente, Série Publicações Técnicas 3, 127p.

DaY, J.W.; C.A.S. Hall; W.M. Kemp \& A. Yáñez-Arancibia. 1989. Estuarine Ecology. New York, Wiley, 558p.

Eskinazi, A.M. 1972. Peixes do Canal de Santa Cruz, Pernambuco, Brasil. Trabalhos Oceanográficos da Universidade Federal de Pernambuco 13: 283-302.

Falcão, M.G.; V. Sarpédonti; H.L. Spach; M.E.B. Otero; G.M.L.N. QueIroz, \& C.A. SANTos. 2006. Ictiofauna em planícies de maré das Baías das Laranjeiras e de Paranaguá, Paraná, Brasil. Revista Brasileira de Zoociências 8 (2): 125-138.

Ferreira, C.E.L. \& J.E.A. Gonçalves. 2006. Community structure and diet of roving herbivorous reef fishes in the Abrolhos Archipelago, south-western Atlantic. Journal of Fish Biology 69 (5): 1533-1551.

Figueiredo, J.L. \& N.A. Menezes. 1978. Manual de peixes marinhos do sudeste do Brasil. II Teleostei. São Paulo, Museu de Zoologia, Universidade de São Paulo, 110p.

Figueiredo, J.L. \& N.A. Menezes. 1980. Manual de peixes marinhos do sudeste do Brasil. III Teleostei. São Paulo, Museu de Zoologia, Universidade de São Paulo, 90p.

Figueiredo, J.L. \& N.A. Menezes. 2000. Manual de peixes marinhos do sudeste do Brasil. VI Teleostei. São Paulo, Museu de Zoologia, Universidade de São Paulo, 116p.

Franco, L. \& K.M.B. Bashirullah. 1992. Alimentación de la lisa (Mugil curema) del Golgo de Cariaco-Estado Suche, Venezuela. Zootecnia Tropical 10 (2): 219-238.

Franks, J.S. \& K.E. VANderKooy. 2000. Feeding habits of juvenile lane snapper Lutjanus synagris from Mississippi coastal waters, with comments on the diet of gray snapper Lutjanus griseus. Gulf and Caribbean Research 12: 11-17.

Freitas, L.E.L.; C.V. Feitosa \& M.E. Araújo. 2006. Mangrove oyster (Crassostrea rhizophorae) (Guilding, 1928) farming areas as artificial reefs for fish: a case study in the state of Ceará, Brazil. Brazilian Journal of Oceanography 54 (1): 31-39.

Froese, R. \& D. Pauly. 2007. FishBase. Available online at: http:/ /www.fishbase.org [Accessed: X/2007].

Furtado, E. 1969. Alimentação de peixes em águas estuarinas do estado do Ceará. Arquivos de Ciências do Mar 9 (2): 111-114.

Garcia, A.M. \& J.P. VieIRA. 2001. O aumento da diversidade de peixes no estuário da Lagoa dos Patos durante o episódio el niño. Atlântica 23: 85-96.

Gay, D.; C. Bassani \& S. Sergipense. 2002. Diel variation and selectivity in the of Cetengraulis edentulus (Cuvier 1828)
(Engraulidae, Clupeiformes) in the Itaipu Lagoon, Niterói, Rio de Janeiro. Atlântica 24 (2): 59-68.

Gibran, F.Z. \& R.M.C. Castro. 1999. Activity, feeding behaviour and diet of Ogcocephalus vespertilio in southern west Atlantic. Journal of Fish Biology 55: 588-595.

Gibson, R.N. \& L. Rовв. 1992. The relationship between body size, sediment grain size and the burying ability of juvenile plaice, Pleuronectes platessa L. Journal of Fish Biology 40: 771-778.

Godefroid, R.S.; H.L. Spach; C. Santos; G. McLaren \& Schwarz-Jr. 2004. Mudanças temporais na abundancia e diversidade da fauna de peixes do infralitoral raso de uma praia do sul do Brasil. Inheringia, Série Zoologia, 94 (1): 95-104.

Grasshoff, K.; M. Ehrhardt \& K. Kremling. 1983. Methods of seawater analysis. Weinheim, Verlag Chemie, 419p.

Guedes, A.P.P.; F.G. Araújo \& M.C.C. Azevedo. 2004. Estratégia trófica dos linguados Citharichthys spilopterus Günther e Symphurus tesselatus (Quoy \& Gaimard) (Actinopterygii, Pleuronectiformes) na Baía de Sepetiba, Rio de Janeiro, Brasil. Revista Brasileira de Zoologia 21 (4): 857-864.

Guedes, D.S.G.; A. L. Vasconcelos-Filho \& R.M. Macedo. 2005. Ictiofauna do infralitoral adjacente às margens do Canal de Santa Cruz - Itapissuma, Pernambuco. Boletim TécnicoCientífico do CEPENE 13 (2): 65-75.

Hahn, N.S.; R. Fugi; V.E. Lourero-Crippa; D. Peretti \& M.R. Russo. 2004. Trophic structure of the fish fauna. p. 139-143. In: A.A. Agostinho; L. Rodrigues; L.C. Gomes; S.M. Thomaz \& L.E. Miranda (Eds). Structure and functioning of the Paraná River and its floodplain. Maringá, EDUEM, 275p.

Köppen, W. 1948. Climatologia: un estudo de los climas de la tierra. Mexico, Fondo de Cultura Economica, 478p.

Labropoulou, M. \& S.K.N. Papadopoulou. 1999. Foraging behaviour patterns of four simpatric demersal fishes. Estuarine, Coastal and Shelf Science 49 (Suppl. A): 99-108.

LACERDA, L.D. 1984. Florestas de beira-mar. Ciência Hoje 3 (13): 62-70.

LiRA, L.; M.C. Zapata \& V.G. Fonseca. 1979. Aspectos da dinâmica do estuário do Rio Formoso, PE. Cadernos Omega da Universidade Federal de Pernambuco 3 (1/2): 133-156.

Livingston, P.A.; D.A. Dwyer; D.L. Wencker; M.S. Yang \& G.M. LANG. 1986. Trophic interactions of key species in the eastern Bering Sea. International North Pacific Fisheries Communication Bulletin. 47: 49-65.

Loebmann, D. \& J.P. Vieira. 2005. Distribuição espacial e abundância das assembléias de peixes no Parque Nacional da Lagoa do Peixe, Rio Grande do Sul, Brasil. Revista Brasileira de Zoologia 22 (3): 667-675.

Lopes, P.R.D. 1999. Nota sobre a alimentação de Albula vulpes (Linnaeus, 1758) (Actinopterygii: Albulidae) na praia de jaguaribe (Ilha de Itamaracá), Pernambuco. Sitientibus 20: 15-22.

Lopes, P.R.D.; J.T. Oliveira-Silva \& A.S.A. Ferreira-Melo. 1998. Contribuição ao conhecimento da ictiofauna do manguezal 
de Cacha Pregos, Ilha de Itaparica, Baía de Todos os Santos, Bahia. Revista Brasileira de Zoologia 15 (2): 315-325.

Lowe-McConnel, R.H. 1999. Estudos ecológicos de comunidades de peixes tropicais. São Paulo, EDUSP, 534p.

Lunardon-Branco, M.J. \& J.O. Branco. 2003. Alimentação natural de Etropus crossotus Jordan \& Gilbert (Teleostei, Pleuronectiformes, Paralichthyidae) na Armação do Itapocoroy, Penha, Santa Catarina, Brasil. Revista Brasileira de Zoologia 20 (4): 631-635.

Macêdo, S.J.; M.J. Flores-Montes \& I.C. Lins. 2000. Características Abióticas da Área, p. 7-25. In: Barros, H.M.; E. EskinazILeÇA; S.J. MACÊdo \& T. LIMA (Eds.) Gerenciamento Participativo de Estuários e Manguezais. Recife, Ed. Universitária da UFPE, 252p.

Macêdo, S.J.; K. Muniz \& M.J. Flores-Montes. 2004. Hidrologia da região costeira e plataforma continental do estado de Pernambuco, p. 255-286. In: E. Eskinazi-Leça; S. NeumannLEITÃo \& M.F. CosTA (Eds).Oceanografia, um cenário tropical. Recife, Bagaço, 761p.

Melo, D.C.P.; M.H.P. Gazineu; J.C. Silva; S.C. Paiva; R.C.P. Rego \& A.A. Salgueiro. 2002. Influência das condições da maré na qualidade da água do rio Formoso. Revista Química \& Tecnologia 1: 20-25.

Menezes, N.A. 1983. Guia prático para conhecimento e identificação das tainhas e paratis (Pisces, Mugilidae) do litoral brasileiro. Revista Brasileira de Zoologia 2 (1): 1-12.

Menezes, N.A. \& J.L. Figueiredo. 1980. Manual de peixes marinhos do sudeste do Brasil. III. Teleostei. São Paulo, Museu de Zoologia, Universidade de São Paulo, 90p.

Menezes, N.A. \& J.L. Figueiredo. 1985. Manual de peixes marinhos do sudeste do Brasil. V. Teleostei. São Paulo, Museu de Zoologia, Universidade de São Paulo, 90p.

Mérona, B. \& J. Rankin-de-Mérona. 2004. Food resource partioning in a fish community of the central Amazon floodplain. Neotropical Ichthyology 2: 75-84.

Monteiro-Neto, C.; C. Blacher; A.A.S. Laurent; F.N. Snizek; M.B. CANozZI \& L. L.C.A. TABAJARA. 1990. Estrutura da comunidade de peixes em águas rasas na região de Laguna, Santa Catarina, Brasil. Atlântica 12 (2): 53-69.

Mullin S.J. 1995. Estuarine fish populations among red mangrove prop roots of small overwash islands. Wetlands 15: 324-329.

Mumby, P.J. 2006. Connectivity of reef fish between mangroves and coral reefs: Algorithms for the design of marine reserves at seascape scales. Biological Conservation 128: 215222.

Nelson, J.S. 1994. Fishes of the World. New York, John Wiley $\&$ Sons, XX+600p.

Paiva, A.C.G.; P.A. Coelho \& M.F.A. Torres. 2005. Influência dos fatores abióticos sobre a macrofauna de substratos inconsolidados da zona entremarés em duas áreas do Canal de Santa Cruz, Pernambuco, Brasil. Arquivos de Ciências do Mar 38: 85-92.
Qasim, S.Z. \& V.N. Sankaranarayanan. 1972. Organic detritus of a tropical estuary. Marine Biology 15 (3): 193-199.

RANDALL, J.E. 1967. Food habits of reef fishes of the West Indies. Studies Tropical Oceanography Miami 5: 665-847.

RANDALL, J.E. 2004. Food habits of reef fishes of the West Indies. Honolulu, Hawaii Institute of Marine Biology University of Hawaii, Honolulu and Bernice P. Bishop Museum, 94p.

Resende, E.K. 2000. Trophic estructure of fish assemblages in the lower Miranda river, Pantanal, Mato Grosso do Sul State. Revista brasileira de Biologia 60 (3): 389-403.

Robertson, A.I. \& S.J.M. Blaber. 1992. Plankton, epibenthos and fish communities, p. 63-100. In: A.I. RoberTson \& D.M AlongI (Eds). Tropical mangrove ecosystems. Washinhton, American Geophysical Union, Coastal and Estuarine Studies 41, 236p.

RoOKer, J.R.; J.P. TURner \& S.A. Holt. 2006. Trophic ecology of Sargassum-associated fishes in the Gulf of Mexico determined from stable isotopes and fatty acids. Marine Ecology Progress Series 313: 249-259.

RozAs, L.P. \& R.J. ZimmERMAn. 2000. Small-scale patterns of nekton use among marsh and adjacent shallow nonvegetated areas of the Galveston Bay Estuary, Texas (USA), Marine Ecology Progress Series 193: 217-239.

SAnTos, F.B. \& R.M.C. CASTRo. 2003. Activity, habitat utilization, feeding behaviour, and diet of the sand moray Gymnothorax ocellatus (Anguilliformes, Muraenidae) in the southwestern Atlantic. Biota Neotropica 3 (1): 1-7.

Saul, A.C.; P.T.M. Cunningham. 1995. Comunidade ictiofaunística da ilha do Bom Abrigo, Cananéia, São Paulo, Brasil. Arquivos de Biologia e Tecnologia 384: 1053-1069.

SAzImA, I. 1983. Scale-eating in characoids and other fishes. Environmental Biology of Fishes 9 (2): 87-101.

SAzIMA, I. 1986. Similarities in feeding behaviour between some marine and freshwater fishes in two tropical communities. Journal of Fish Biology 29: 53-65.

Sierra, L.M.; R. Claro \& O.A. Popova. 1994. Alimentacion y relaciones tróficas, p. 263-284. In: R. Claro (Ed.). Ecología de los Peces Marinos de Cuba. Mexico, Instituto de Oceanología Academia de Ciencias de Cuba y Centro de Investigaciones de Quintana Roo, 545p.

Silva, M.H.; J.Z.O. Passavante; M.G.G. Silva-Cunha; D.A.N. Vieira; C.K.S. Grego \& K. MunIz. 2004. Distribuição espacial e sazonal da biomassa fitoplanctônica e dos parâmetros hidrológicos no estuário do rio Formoso (Rio formoso, Pernambuco, Brasil). Tropical Oceanography 32 (1): 89106.

Soares, L.S.H.; M.A. Gasalla; M.A.T. Rios; M.V. Arrasa \& C.L.B. Rossi-Wongtschowski. 1993. Grupos tróficos de onze espécies dominantes de peixes demersais da plataforma continental interna de Ubatuba, Brasil. Publicação Especial do Instituto Oceanográfico de São Paulo 10: 189-198.

Spach, H.L.; C. Santos \& R.S. Godefroid. 2003. Padrões tempo- 
rais na assembléia de peixes na gamboa do Sucuriú, Baía de Paranaguá, Brasil. Revista Brasileira de Zoologia 20 (4): 591-600.

Spach, H.L.; C. Santos; R. S. Godefroid; M. Nardi \& F. Cunha. 2004. A study of the fish community structure in a tidal creek. Brazilian Journal Biology 64 (2): 337-351.

Suguio, K. 1973. Introdução à sedimentologia. São Paulo, Blucher/EDUSP, 312p.

TeixeIRA, R.L. 1994. Abundance, reproductive period, and feeding habits of eleotrid fishes in estuarine habitats of north-east Brazil. Journal of Fish Biology 45: 749-761.

Teixeira, T.P.; B.C.T. Pinto; B.F. Terra; E.O. Estiliano; D. Gracia \& F.G. ARAújo. 2005. Diversidade das assembléias de peixes nas quatro unidades geográficas do rio Paraíba do Sul. Iheringia, Série Zoologia, 95 (4): 347-357.

U.S. Naval Oceanographic Office. 1965. Oceanographic Atlas of the North Atlantic Ocean. Washington, U.S. Naval Oceanographic Office, Section 5, Marine Geology, 79p

Vasconcelos-Filho, A.L.; K.C. DA Silva \& F.D. Acioli. 1998. Hábitos alimentares de Sphoeroides testudineus (Linnaeus, 1758) (Teleostei: Tetraodontidae) no Canal de Santa Cruz, Itamaracá - PE. Trabalhos Oceanográficos da Universidade Federal de Pernambuco 26 (1): 145-157.

Vasconcelos-Filho, A.L.; S. Neuman-Leitão; E. Eskinazi-LeÇa; R.
Schwamborn; A.M.E. Oliveira \& M.N. Paranaguá. 2003. Trophic interactions between fish and other compartment communities in a tropical estuary in Brazil as indicator of environmental quality, p. 173-183. In: E. TiezzI; C.A. BREBBIA \& J.L. USÓ (Eds). Ecosystems and Sustainable Development IV. Boston, Southampton, 772p.

VIDY, G. 2000. Estuarine and mangrove systems and the nursery concept: which is which? The case of the Sine-Saloum system (Senegal). Wetlands Ecology and Management 8: 37-51.

Villarroel, P.R. 1994. Estrutura de las comunidades de peces de la Laguna de Raya, isla de Margrita, Venezuela. Ciencias Marinas 20 (1): 1-16.

Whitehead, P.J.P.; G.J. Nelson \& T. Wongratana. 1988. FAO species catalogue. 7. Clupeoid fishes of the world (Suborder Clupeoidei). An annotated and illustrated catalogue of the herrings, sardines, pilchards, sprats, shads, anchovies and wolf-herrings. Part 2 - Engraulididae. FAO Fisheries Synopsis. 125 (7/2): 305-579.

Zahorcsak, P.; R.A. Silvano \& I. Sazima. 2000. Feeding biology of a guild of benthivorous fishes in a sandy shore on southeastern brazilian coast. Revista Brasileira de Biologia 60 (3): 511-518.

ZAVALA-CAMIN, L.A. 1996. Introdução aos estudos sobre alimentação natural em peixes. Maringá, Nupélia, 127p.

Submitted: 23.I.2008; Accepted: 27.XI.2008.

Editorial responsibility: Paulo da Cunha Lana 